1 Hacettepe Journal of Mathematics and Statistics

$\bigcap$ Volume 45 (5) (2016), 1335-1342

\title{
On rings over which every finitely generated module is a direct sum of cyclic modules
}

\author{
M. Behboodi* ${ }^{* \dagger}$ and G. Behboodi Eskandari*
}

\begin{abstract}
In this paper we study (non-commutative) rings $R$ over which every finitely generated left module is a direct sum of cyclic modules (called left FGC-rings). The commutative case was a well-known problem studied and solved in 1970 s by various authors. It is shown that a Noetherian local left FGC-ring is either an Artinian principal left ideal ring, or an Artinian principal right ideal ring, or a prime ring over which every two-sided ideal is principal as a left and a right ideal. In particular, it is shown that a Noetherian local duo-ring $R$ is a left FGCring if and only if $R$ is a right FGC-ring, if and only if, $R$ is a principal ideal ring.
\end{abstract}

Keywords: Cyclic modules; FGC-rings; duo-rings; principal ideal rings. 2000 AMS Classification: Primary 16D10, Secondary 16D70, 16P20, 16N60

Received: 01.11.2013 Accepted : 03.03.2014 Doi : 10.15672/HJMS.2015449425

\section{Introduction}

The question of which rings have the property that every finitely generated module is a direct sum of cyclic modules has been around for many years. We will call these rings FGC-rings. The problem originated in I. Kaplansky's papers [13] and [14], in which it was shown that a commutative local domain is FGC if and only if it is an almost maximal valuation ring. For several years, this is one of the major open problems in the theory. R. S. Pierce [19] showed that the only commutative FGC-rings among the commutative (von Neumann) regular rings are the finite products of fields. A deep and difficult study was made by Brandal [3], Shores-R. Wiegand [22], S. Wiegand [24], Brandal-R. Wiegand [4] and Vámos [23], leading to a complete solution of the problem in the commutative case. To show that a commutative FGC-ring cannot have an infinite number of minimal

*Department of Mathematical Sciences, Isfahan University of Technology, P.O.Box: 8415683111, Isfahan, Iran and School of Mathematics, Institute for Research in Fundamental Sciences, P.O.Box: 19395-5746, Tehran, Iran Emails: mbehbood@cc.iut.ac.ir, gh.behboodi@ipm.ir.

${ }^{\dagger}$ The research of this author was in part supported by a grant from IPM (No. 92130413). This research is partially carried out in the IPM-Isfahan Branch. 
prime ideals required the study of topological properties (so-called Zariski and patch topologies). For complete and more leisurely treatment of this subject, see Brandal [2]. It gives a clear and detailed exposition for the reader wanting to study the subject. The main result reads as follows: A commutative ring $R$ is an FGC-ring exactly if it is a finite direct sum of commutative rings of the following kinds: (i) maximal valuation rings; (ii) almost maximal Bézout domains; (iii) so-called torch rings (see [2] or [8] for more details on the torch rings).

The corresponding problem in the non-commutative case is still open; see [21, Appendix B. Dniester Notebook: Unsolved Problems in the Theory of Rings and Modules. Pages 461-516] in which the following problem is considered.

Problem (I. Kaplansky, reported by A. A. Tuganbaev [21, Problem 2.45]): Describe the rings in which every one-sided ideal is two-sided and over which every finitely generated module can be decomposed as a direct sum of cyclic modules.

Through this paper, all rings have identity elements and all modules are unital. A left $F G C$-ring is a ring $R$ such that each finitely generated left $R$-module is a direct sum of cyclic submodules. A right FGC-ring is defined similarly, by replacing the word left with right above. A ring $R$ is called a $F G C$-ring if it is a both left and right FGC-ring. Also, a ring $R$ is called duo-ring if each one-sided ideal of $R$ is two-sided. Therefore, the Kaplansky problem is: Describe the FGC-duo-rings.

In this paper we investigate Noetherian local left FGC-rings (see Section 2). Also, we will present a partial solution to the above problem of Kaplansky (see Section 3).

\section{On left FGC-rings}

A ring $R$ is local in case $R$ has a unique left maximal ideal. An Artinian (resp. Noetherian) ring is a ring which is both left and right Artinian (resp. Noetherian). A principal ideal ring is a ring which is both left and right principal ideal ring. Also, for a subset $S$ of ${ }_{R} M$, we denote by $\operatorname{Ann}_{R}(S)$, the annihilator of $S$ in $R$. A left $R$-module $M$ which has a composition series is called a module of finite length. The length of a composition series of ${ }_{R} M$ is said to be the length of ${ }_{R} M$ and denoted by $\operatorname{length}\left({ }_{R} M\right)$.

We begin with the following lemma which is an associative, non-commutative version of Brandal [2, Proposition 4.3] for local rings $(R, \mathcal{M})$ with $\mathcal{M}^{2}=(0)$. Also, the proof is based on a slight modification of the proof of [1, Theorem 3.1].

2.1. Lemma. Let $(R, \mathcal{M})$ be a local ring with $\mathcal{M}^{2}=(0)$ and ${ }_{R} \mathcal{M}=R y_{1} \oplus \ldots \oplus R y_{t}$ such that $t \geq 2$ and each $R y_{i}$ is a minimal left ideal of $R$. If there exist $0 \neq x_{1}, x_{2} \in \mathcal{M}$ such that $x_{1} R \cap x_{2} R=(0)$, then the left $R$-module $(R \oplus R) / R\left(x_{1}, x_{2}\right)$ is not a direct sum of cyclic modules.

Proof. Since ${ }_{R} \mathcal{M}=R y_{1} \oplus \ldots \oplus R y_{t}$ and each $R y_{i}$ is a minimal left ideal of $R$, we conclude that $R$ is of finite composition length and length $\left({ }_{R} R\right)=t+1$. We put ${ }_{R} G=(R \oplus$ $R) / R\left(x_{1}, x_{2}\right)$. Since $x_{1}, x_{2} \in \mathcal{M}$ and $\mathcal{M}^{2}=(0)$, we conclude that $\operatorname{Ann}_{R}\left(R\left(x_{1}, x_{2}\right)\right)=\mathcal{M}$. Thus $R\left(x_{1}, x_{2}\right)$ is simple and hence

$$
\operatorname{length}\left({ }_{R} G\right)=2 \times \operatorname{length}\left({ }_{R} R\right)-\operatorname{length}\left({ }_{R} R\left(x_{1}, x_{2}\right)\right)=2(t+1)-1 .
$$

We claim that every non-zero cyclic submodule $R z$ of $G$ has length 1 or $t+1$. If $\mathcal{M} z=0$, then length $(R z)=1$ since $R z \simeq R / \mathcal{M}$. Suppose that $\mathcal{M} z \neq 0$, then there exist $c_{1}, c_{2} \in R$ such that $z=\left(c_{1}, c_{2}\right)+R\left(x_{1}, x_{2}\right)$. If $c_{1}, c_{2} \in \mathcal{M}$, then $\mathcal{M} z=0$, since $\mathcal{M}^{2}=0$. Thus without loss of generality, we can assume that $z=\left(1, c_{2}\right)+R\left(x_{1}, x_{2}\right)$ (since if $c_{1} \notin \mathcal{M}$, then $c_{1}$ is unit). Now let $r \in \operatorname{Ann}_{R}(z)$, then $r\left(1, c_{2}\right)=t\left(x_{1}, x_{2}\right)$ for some $t \in R$. It follows that $r=t x_{1}$ and $r c_{2}=t x_{2}$. Thus $t x_{2}=t x_{1} c_{2}$. If $t \notin \mathcal{M}$, then $t$ is unit and so $x_{2}=x_{1} c_{2}$ 
that it is contradiction ( since $x_{1} R \cap x_{2} R=(0)$ ). Thus $t \in \mathcal{M}$ and so $r=t x_{1}=0$. Therefore, $\operatorname{Ann}_{R}(z)=0$ and so $R z \cong R$. It follows that length $(R z)=t+1$.

Now suppose the assertion of the lemma is false. Then ${ }_{R} G$ is a direct sum of cyclic modules and since ${ }_{R} G$ is of finite length, we have

$$
G=R w_{1} \oplus \ldots \oplus R w_{k} \oplus R v_{1} \oplus \ldots \oplus R v_{l},
$$

where $l, k \geq 0$, and each $R w_{i}$ is of length $t+1$ and each $R v_{j}$ is of length 1 . Clearly $\mathcal{M} \oplus \mathcal{M}$ is not a simple left $R$-module. Since $R\left(x_{1}, x_{2}\right)$ is simple, $\mathcal{M} G=(\mathcal{M} \oplus \mathcal{M}) / R\left(x_{1}, x_{2}\right) \neq 0$. It follows that $k \geq 1$. Also, length $\left({ }_{R} G\right)=2(t+1)-1=k(t+1)+l$ and this implies that $k=1$ and $l=t$. Since $\mathcal{M} v_{i}=0$ for each $i, \mathcal{M} G=\mathcal{M} w_{1}$ and hence

$$
G / \mathcal{M} G \simeq R w_{1} / \mathcal{N} w_{1} \oplus R v_{1} \oplus \ldots \oplus R v_{t} .
$$

It follows that $\operatorname{length}\left({ }_{R} G / \mathcal{M} G\right)=1+t$. On the other hand, we have

$$
G / \mathcal{M} G \cong R / \mathcal{M} \oplus R / \mathcal{M}
$$

and so length $\left({ }_{R} G / \mathcal{M} G\right)=2$ and so $t=1$, a contradiction. Thus the left $R$-module $(R \oplus R) / R\left(x_{1}, x_{2}\right)$ is not a direct sum of cyclic modules.

We recall that the $\operatorname{socle} \operatorname{soc}(R M)$ of a left module $M$ over a ring $R$ is defined to be the sum of all simple submodules of $M$.

2.2. Theorem. Let $(R, \mathcal{M})$ be a local ring such that ${ }_{R} \mathcal{M}$ and $\mathcal{M}_{R}$ are finitely generated. If every left $R$-module with two generators is a direct sum of cyclic modules, then either $\mathcal{M}$ is a principal left ideal or $\mathcal{M}$ is a principal right ideal.

Proof. We can assume that $\mathcal{M}$ is not a principal left ideal of $R$. One can easily see that $\mathcal{M}_{R}$ is generated by $\left\{x_{1}, \cdots, x_{n}\right\}$ if and only if $\mathcal{M} / \mathcal{M}^{2}$ is generated by the set $\left\{x_{1}+\mathcal{M}^{2}, \cdots, x_{n}+\mathcal{M}^{2}\right\}$ as a right ideal of $R / \mathcal{M}^{2}$. Thus it suffices to show that $\mathcal{M} / \mathcal{M}^{2}$ is a principal right ideal of $R / \mathcal{M}^{2}$. Since every left $R$-module with two generators is a direct sum of cyclic modules, we conclude that every left $R / \mathcal{M}^{2}$-module with two generators is also a direct sum of cyclic modules. Therefore, without loss of generality we can assume that $\mathcal{M}^{2}=(0)$. It follows that $\operatorname{soc}\left({ }_{R} R\right)=\operatorname{soc}\left(R_{R}\right)=\mathcal{M}$. Since ${ }_{R} \mathcal{M}$ is finitely generated, ${ }_{R} \mathcal{M}=R y_{1} \oplus \ldots \oplus R y_{t}$ such that $t \geq 2$ and each $R y_{i}$ is a minimal left ideal of $R$. We claim that $\mathcal{M}_{R}=x R$, for if not, then we can assume that $\mathcal{M}_{R}=\oplus_{i \in I} x_{i} R$ where $|I| \geq 2$ and each $x_{i} R$ is a minimal right ideal of $R$. We can assume that $\{1,2\} \subseteq I$ and so $0 \neq x_{1}$, $x_{2} \in \mathcal{M}$ and $x_{1} R \cap x_{2} R=(0)$. Now by Lemma 2.1 , the left $R$-module $(R \oplus R) / R\left(x_{1}, x_{2}\right)$ is not a direct sum of cyclic modules, a contradiction. Thus $\mathcal{M}$ is principal as a right ideal of $R$.

A ring whose lattice of left ideals is linearly ordered under inclusion, is called a left uniserial ring. A uniserial ring is a ring which is both left and right uniserial. Note that left and right uniserial rings are in particular local rings and commutative uniserial rings are also known as valuation rings.

Next, we need the following lemma from [18].

2.3. Lemma. (See Nicholson and Sánchez-Campos [18, Theorem 9]) For any ring $R$, the following statements are equivalent:

(1) $R$ is local, $J(R)=R x$ for some $x \in R$ and $x^{k}=0$ for some $k \in \mathbb{N}$.

(2) There exist $x \in R$ and $k \in \mathbb{N}$ such that $x^{k-1} \neq 0$ and

$$
R \supset R x \supset \ldots \supset R x^{k}=(0)
$$

are the only left ideals of $R$.

(3) $R$ is left uniserial of finite composition length. 
2.4. Theorem. Let $(R, \mathcal{M})$ be a local ring such that ${ }_{R} \mathcal{M}$ and $\mathcal{M}_{R}$ are finitely generated and $\mathcal{M}^{k}=(0)$ for some $k \in \mathbb{N}$. If every left $R$-module with two generators is a direct sum of cyclic modules, then either $R$ is a left Artinian principal left ideal ring or $R$ is a right Artinian principal right ideal ring.

Proof. Assume that every left $R$-module with two generators is a direct sum of cyclic modules. Then by Theorem 2.2, either $\mathcal{N}$ is a principal left ideal or $\mathcal{M}$ is a principal right ideal. If $\mathcal{M}$ is a principal left ideal, then by Lemma 2.3, $R$ is a left Artinian principal left ideal ring. Thus we can assume that $\mathcal{M}$ is a principal right ideal. Then by using Lemma 2.3 to the right side, $R$ is a right Artinian principal right ideal ring.

Next, we need the following lemma from Mohamed H. Fahmy-Susan Fahmy[9]. We note that their definition of a local ring is slightly different than ours; they defined a local ring (resp. scalar local ring) as a ring $R$ such that it contains a unique maximal ideal $\mathcal{M}$ and $R / \mathcal{M}$ is an Artinian ring (resp. division ring). Thus our definition of a local ring and the scalar local ring coincide.

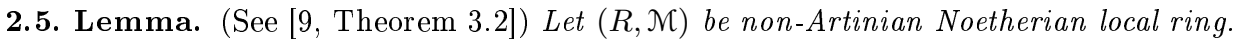
Then the following conditions are equivalent:

(1) $\mathcal{M}$ is principal as a right ideal.

(2) $\mathcal{M}$ is principal as a left ideal.

(3) Every two-sided ideal of $R$ is principal as a left ideal.

(4) Every two-sided ideal of $R$ is principal as a right ideal. Moreover, $R$ is a prime ring.

Now we are in a position to prove the main theorem of this section.

2.6. Theorem. Let $(R, \mathcal{M})$ be a Noetherian local ring. If every left $R$-module with two generators is a direct sum of cyclic modules, then one of the following holds:

(1) $R$ is an Artinian principal left ideal ring.

(2) $R$ is an Artinian principal right ideal ring.

(3) $R$ is a prime ring and every two-sided ideal of $R$ is principal as both left and right ideals.

Proof. First we assume that $R$ is an Artinian ring. Thus by Theorem 2.4, either $R$ is an Artinian principal left ideal ring or $R$ is an Artinian principal right ideal ring. Now we assume that $R$ is not an Artinian ring. By Theorem 2.2, either $\mathcal{M}$ is a principal left ideal or $\mathcal{M}$ is a principal right ideal. Thus by Lemma $2.5, R$ is a prime ring and every two-sided ideal of $R$ is principal as both left and right ideals.

\section{A partial solution of Kaplansky's problem on duo-rings}

A ring $R$ is said to be left (resp. right) hereditary if every left (resp. right) ideal of $R$ is projective as a left (resp. right) $R$-module. If $R$ is both left and right hereditary, we say that $R$ is hereditary. Recall that a PID is a domain $R$ in which any left and any right ideal of $R$ is principal. Clearly, any PID is hereditary.

Let $R$ be an hereditary prime ring with quotient $\operatorname{ring} Q$ and $A$ be a left $R$-module. Following Levy [17], we say that $a \in A$ is a torsion element if there is a regular element $r \in R$ such that $r a=0$. Since, by Goldie's theorem, $R$ satisfies the Ore condition, the set of torsion elements of $A$ is a submodule $t(A) \subseteq A$. $A / t(A)$ is evidently torsion free (has no torsion elements). 
3.1. Lemma. (Eisenbud-Robson [6, Theorem 2.1]) Let $R$ be an hereditary Noetherian prime ring, and let $A$ be a finitely generated left $R$-module. Then $A / t(A)$ is projective and $A \cong t(A) \oplus A / t(A)$.

A Dedekind prime ring [20] is an hereditary Noetherian prime ring with no proper idempotent two-sided ideals (see [7]). Clearly if a duo-ring $R$ is a PID, then $R$ is a Dedekind prime ring.

3.2. Lemma. (Eisenbud-Robson [6, Theorem 3.11]) Let $R$ be a Dedekind prime ring. Then every finitely generated torsion left $R$-module $A$ is a direct sum of cyclic modules.

3.3. Lemma. (Eisenbud-Robson [6, Theorem 2.4]) Let $R$ be a Dedekind prime ring, and let $A$ be a projective left $R$-module. Then:

(1) If $A$ is finitely generated, then $A \cong F \oplus I$ where $F$ is a finitely generated free module and $I$ is a left ideal of $R$.

(2) If $A$ is not finitely generated, then $A$ is free.

3.4. Proposition. Let $R$ be a Dedekind prime ring. If $R$ is a left principal ideal ring, then $R$ is a left FGC-ring.

Proof. Suppose that $A$ is a finitely generated left $R$-module. Since $R$ is a Dedekind prime ring, $R$ is Noetherian and so $A$ is also a Noetherian left $R$-module. Thus by Lemma 3.1, $A / t(A)$ is projective and $A \cong t(A) \oplus A / t(A)$. By Lemma 3.2, $t(A)$ is a direct sum of cyclic modules. Also by Lemma 3.3, $A / t(A) \cong F \oplus I$ where $F$ is a free module and $I$ is a left ideal of $R$. Since $R$ is a principal left ideal ring, $I$ is a cyclic left $R$-module, i.e., $A / t(A)$ is a direct sum of cyclic modules. Thus, $A \cong t(A) \oplus A / t(A)$ is a direct sum of cyclic modules. Therefore, $R$ is a left FGC-ring.

The following proposition is an answer to the question: "What is the structure of FGC Noetherian prime duo-rings?"

3.5. Proposition. (See also Jacobson [11, Page 44, Theorems 18 and 19]) Let $R$ be a Noetherian prime duo-ring (i.e., $R$ is a Noetherian duo-domain). Then the following statements are equivalents:

(1) $R$ is an FGC-ring.

(2) $R$ is a left FGC-ring.

(3) $R$ is a principal ideal ring.

The same characterizations also apply for right $R$-modules.

Proof. $(1) \Rightarrow(2)$ is clear. $(2) \Rightarrow(3)$. Suppose that $I$ is an ideal of $R$. Since $I$ is a direct sum of principal ideals of $R$ and $R$ is a domain, we conclude that $I$ is principal. Thus, $R$ is a principle ideal ring. $(3) \Rightarrow(1)$ is by Proposition 3.4 .

A left (resp., right) Köthe ring is a ring $R$ such that each left (resp., right) $R$-module is a direct sum of cyclic submodules. A ring $R$ is called a Köthe ring if it is a both left and right Köthe ring. In [16] Köthe proved that an Artinian principal ideal ring is a Köthe ring. Furthermore, a commutative ring $R$ is a Köthe ring if and only if $R$ is an Artinian principal ideal ring (see Cohen and Kaplansky [5]). The corresponding problem in the non-commutative case is still open (see [21, Appendix B, Problem 2.48] or Jain-Srivastava [12, Page 40, Problem 1]. Recently, a generalization of the Köthe-CohenKaplansky theorem is given in [1]. In fact: in [1, Corollary 3.3.], it is shown that if $R$ is a ring in which all idempotents are central, then $R$ is a Köthe ring if and only if $R$ is an Artinian principal ideal ring. 
Next, the following theorem is an answer to the question: "What is the structure of FGC Noetherian local duo-rings?"

3.6. Theorem. Let $(R, \mathcal{M})$ be a Noetherian local duo-ring. Then the following statements are equivalent:

(1) $R$ is an FGC-ring.

(2) $R$ is a left $F G C$-ring.

(3) Every left $R$-module with two generators is a direct sum of cyclic modules.

(4) Either $R$ is an Artinian principal ideal ring or $R$ is a principal ideal domain.

(5) $R$ is a principal ideal ring.

The same characterizations also apply for right $R$-modules.

Proof. $(1) \Rightarrow(2) \Rightarrow(3)$ is clear.

$(3) \Rightarrow(4)$. Suppose that every left $R$-module with two generators is a direct sum of cyclic modules. Thus by Theorem $2.2, \mathcal{M}$ is principal as both left and right ideals. If $R$ is Artinian, then by Theorem 2.4, $R$ is an Artinian principal ideal ring. If $R$ is not Artinian, then by Lemma $2.5, R$ is a principal ideal domain.

$(4) \Rightarrow(1)$. If $R$ is an Artinian principal ideal ring, then by the Köthe result, each left, and each right $R$-module is a direct sum of cyclic modules. Thus $R$ is an FGC-ring. Now assume that $R$ is a principal ideal domain. Then by Proposition 3.5, $R$ is an FGC-ring. $(4) \Rightarrow(5)$ is clear.

$(5) \Rightarrow(4)$. Assume that $R$ is a principal ideal ring. Then $\mathcal{M}$ is principal as both left and right ideals. If $R$ is Artinian, then by Lemma 2.3, $R$ is an Artinian principal ideal ring. If $R$ is not Artinian, then by Lemma 2.5, $R$ is a principal ideal domain.

Let $R=\prod_{i=1}^{n} R_{i}$ be a finite product of rings $R_{i}$. Clearly $R$ is a principal ideal ring if and only if each $R_{i}$ is a principal ideal ring. On the other hand if $R$ is a left FGC-ring, then each $R_{i}$ is also a left FGC-ring. Thus as a corollary of Proposition 3.5 and Theorem 3.6, we have the following result.

3.7. Corollary. Let $R=\prod_{i=1}^{n} R_{i}$ be a finite product of Noetherian duo-rings $R_{i}$ such that each $R_{i}$ is a domain or a local ring. Then the following statements are equivalent:

(1) $R$ is an $F G C$-ring.

(2) $R$ is a left $F G C$-ring.

(3) $R$ is a principal ideal ring.

The same characterizations also apply for right $R$-modules.

Next, we need the following lemma from [10] about Artinian duo-rings (its proof is worthwhile even in the commutative case (see [10, Corollary 4] or [15, Lemma 4.2])

3.8. Lemma. Let $R$ be an Artinian duo-ring. Then $R$ is a finite direct product of Artinian local duo rings.

Next, we give the following characterizations of an Artinian FGC duo-ring. In fact, on Artinian duo-rings, the notions "FGC" and "Köthe" coincide.

3.9. Theorem. Let $R$ be an Artinian Duo-ring. Then the following statements are equivalent:

(1) $R$ is a left FGC-ring.

(2) $R$ is an FGC-ring.

(3) Every left $R$-module with two generators is a direct sum of cyclic modules.

(4) $R$ is a left Köthe-ring.

(5) $R$ is a Köthe-ring.

(6) $R$ is a principal ideal ring. 
The same characterizations also apply for right $R$-modules.

Proof. Since $R$ is an Artinian duo-ring, by Lemma 3.8, $R=\prod_{i=1}^{n} R_{i}$ such that each $R_{i}$ is an Artinian local duo-ring. Thus by the Köthe result and Corollary 3.7, the proof is complete.

Acknowledgments. The authors would like to thank the anonymous referee for a careful checking of the details and for helpful comments that improved this paper.

\section{References}

[1] Behboodi, M., Ghorbani, A., Moradzadeh-Dehkordi A. and Shojaee, S. H. On left Köthe rings and a generalization of a Köthe-Cohen-Kaplansky theorem, Proc. Amer. Math. Soc. 142., no. 8, 2625-2631, (2014).

[2] Brandal, W. Commutative Rings Whose Finitely Generated Modules decompose, Lecture Notes in Mathematics, Vol. 723. (Springer, 1979).

[3] Brandal, W. Almost maximal integral domains and finitely generated modules. Trans. Amer. Math. Soc. 183., 203-222, (1973).

[4] Brandal, W., and Wiegand, R. Reduced rings whose finitely generated modules decompose. Comm. Algebra 6., (2) 195-201, (1978).

[5] Cohen, I. S. and Kaplansky, I. Rings for which every module is a direct sum of cyclic modules, Math. Z. 54., 97-101, (1951).

[6] Eisenbud, D. and Robson, J. C. Modules over Dedekind prime rings, J. Algebra 16., 67-85 (1970).

[7] Eisenbud, D. and Robson, J. C. Hereditary Noetherian prime rings, J. Algebra 16., 86-104, (1970).

[8] Facchini, A. On the structure of torch rings. Rocky Mountain J. Math. 13., (3) 423-428, (1983).

[9] Fahmy, M. H. and Fahmy, S. On non-commutative noetherian local rings, non-commutative geometry and particle physics, Chaos, Solitons and Fractals 14., 1353-1359, (2002).

[10] Habeb, J. M. A note on zero commutative and duo rings, Math. J. Okayama Univ 32., 73-76, (1990).

[11] Jacobson, N. The Theorey of Rings, Amerian Mathematical Society Mathematical Surveys, vol. I, (American Mathematical Society, New York, 1943).

[12] Jain, S. K. and Srivastava, Ashish K. Rings characterized by their cyclic modules and right ideals: A survey-I. (See http://euler.slu.edu/ srivastava/articles.html).

[13] Kaplansky, I. Elementary divisors and modules. Trans. Amer. Math. Soc. 66., 464-491, (1949).

[14] Kaplansky, I. Modules over Dedekind rings and valuation rings. Trans. Amer. Math. Soc. 72., 327-340, (1952).

[15] Karamzadeh, N. S. and Karamzadeh, O. A. S. On artinian modules over Duo rings, Comm. Algebra 38., 3521-3531, (2010).

[16] Köthe, G. Verallgemeinerte abelsche gruppen mit hyperkomplexem operatorenring, Math. Z. 39., 31-44, (1935).

[17] Levy, L. Torsion-Free and divisible modules over non-integral domains, Canad. J. Math. 15., 132-151, (1963).

[18] Nicholson, W. K. and Sánchez-Campos, E. Rings with the dual of the isomorphism theorem, J. Algebra 271., (1) 391-406, (2004).

[19] Pierce, R. S. Modules over commutative regular rings. Mem. Amer. Math. Soc. 70., (1967).

[20] Robson, J. C. Non-commutative Dedekind rings, J. Algebra 9., 249-265, (1968).

[21] Sabinin, L., Sbitneva, L. and Shestakov, I. Non-associative algebra and its applications, Lecture Notes in Pure and Applied Mathematics 246., Chapman and Hall/CRC, (2006).

[22] Shores, T. S. and Wiegand, R. Rings whose finitely generated modules are direct sums of cyclics. J. Algebra 32., 152-172, (1974).

[23] Vámos, P. The decomposition of finitely generated modules and fractionally self-injective rings. J. London Math. Soc. 16., 209-220, (1977). 
[24] Wiegand, R. and Wiegand, S. Commutative rings whose finitely generated modules are direct sums of cyclics. Abelian group theory (Proc. Second New Mexico State Univ. Conf., Las Cruces, N.M., 1976), pp. 406-423. Lecture Notes in Math., Vol. 616., (Springer, Berlin, 1977). 